University of Nebraska - Lincoln

DigitalCommons@University of Nebraska - Lincoln

3-1995

\title{
Analyzing Correlations of Three Types in Selected Lines of Drosophila melanogaster That Have Evolved Stable Extreme Geotactic Performance
}

\author{
Scott F. Stoltenberg \\ University of Illinois at Urbana-Champaign, sstoltenberg2@unl.edu \\ Jerry Hirsch \\ University of Illinois at Urbana-Champaign \\ Stewart H. Berlocher \\ University of Illinois at Urbana-Champaign, stewartb@illinois.edu
}

Follow this and additional works at: https://digitalcommons.unl.edu/psychfacpub

Part of the Entomology Commons, Genetics Commons, and the Psychology Commons

Stoltenberg, Scott F.; Hirsch, Jerry; and Berlocher, Stewart H., "Analyzing Correlations of Three Types in Selected Lines of Drosophila melanogaster That Have Evolved Stable Extreme Geotactic Performance" (1995). Faculty Publications, Department of Psychology. 1054.

https://digitalcommons.unl.edu/psychfacpub/1054

This Article is brought to you for free and open access by the Psychology, Department of at DigitalCommons@University of Nebraska - Lincoln. It has been accepted for inclusion in Faculty Publications, Department of Psychology by an authorized administrator of DigitalCommons@University of Nebraska - Lincoln. 


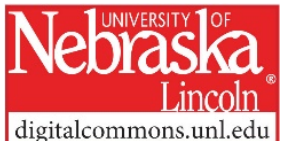

Published in Journal of Comparative Psychology 109:1 (March 1995), pp. 85-94; doi: 10.1037/0735-

7036.109.1.85

Copyright (C 1995 by the American Psychological Association, Inc. Used by permission.

Submitted April 11, 1994; revised August 31, 1994; accepted September 1, 1994.

\title{
Analyzing Correlations of Three Types in Selected Lines of Drosophila melanogaster That Have Evolved Stable Extreme Geotactic Performance
}

\author{
Scott F. Stoltenberg, ${ }^{1}$ Jerry Hirsch, ${ }^{2}$ and Stewart H. Berlocher ${ }^{3}$
}

1. Department of Psychology, University of Illinois at Urbana-Champaign, Champaign, Illinois, USA

2. Department of Psychology and Department of Ecology, Ethology, and Evolution, University of Illinois at Urbana-Champaign, Champaign, Illinois, USA

3. Department of Entomology, University of Illinois at Urbana- Champaign, Champaign, Illinois, USA

Corresponding author - Jerry Hirsch, Department of Psychology, University of Illinois, 603 East Daniel Street, Champaign, IL 61820, USA, email jhirsch@uiuc.edu

\begin{abstract}
The behavior-genetic analysis of Drosophila melanogaster with geotactic performance as the phenotype is an ideal model system with which to investigate the complex relations between heredity and behavior. As part of a long-term, 38-year study, we report 4 experiments that identify and analyze trait correlations in the selected high- and low-geotaxis lines. We performed $\mathrm{F}_{2}$ correlational analyses and backcrosses to examine 3 types of correlations: (a) genotype-genotype (alcohol dehydrogenase [Adh]-amylase [Amy]), (b) genotype-phenotype (Adh and Amy-geotaxis), and (c) phenotype-phenotype (mate preference-geotaxis). Only the $A d h$-geotaxis correlation survived meiosis and reappeared in the $\mathrm{F}_{2}$ generation, which indicates a genotype-phenotype correlation, whereas the others did not. The importance of hybrid correlational analysis to the behavior-genetic analysis of a species is discussed.
\end{abstract}

Trait correlations are basic to behavior-genetic analysis, especially when selected or inbred lines are analyzed. Making sense of such correlations is one of the most challenging tasks that face investigators because many correlations appear but only some can be interpreted. 
Three types of trait correlations are possible: genotype-genotype, genotype-phenotype, and phenotype-phenotype. Genotype-genotype correlations have also been referred to as linkage disequilibria or gametic phase disequilibria (Falconer, 1989). Such correlations have been interpreted as evidence of coadapted gene complexes and are of considerable evolutionary interest. Genotype-phenotype correlations are those of greatest interest to behavior-genetic analysts because of their potential value for understanding heredity - behavior relations. Phenotype-phenotype correlations are generally the most obvious of the three types of correlations in selected or inbred lines because phenotypes are usually easier to assess than genotypes.

The method of hybrid correlational analysis permits analyzing trait correlations by taking advantage of Mendelian processes that allow traits influenced by genetically independent systems to be dissociated (see McCleara, 1967; Tully, Zawistowski, \& Hirsch, 1982). This method can be used when two traits are correlated in different interfertile populations. For example, in Line 1, high scores on Trait A may be associated with high scores on Trait B, and in Line 2, low scores on Trait A may be associated with low scores on Trait B. Thus, Traits A and B are positively correlated in Lines 1 and 2 (hybrid correlational analysis can also be used with negative correlations). Individuals from Lines 1 and 2 are crossed to produce $F_{1}$ generation progeny, which are subsequently intermated to produce $F_{2}$ generation progeny, which are tested for both traits.

Logically, three outcomes are possible. (a) The trait correlation may persist at a magnitude similar to that observed in the parental lines. This suggests that the two traits are influenced by the same genetic system (i.e., pleiotropy). (b) The trait correlation may be significantly attenuated. This result indicates that either the two traits are influenced by genetic systems that overlap to some extent but are not identical or that genes influencing the two traits are on the same chromosome (i.e., linkage). (c) The trait correlation may be eliminated at independent assortment during meiosis in the $\mathrm{F}_{1}$ generation. This suggests that the correlation between Traits A and B in the parental lines is the result of neither pleiotropy nor correlated genetic systems.

There are, of course, other ways to analyze trait correlations. The use of replicate selection lines has been supported by several authors (Crabbe, Phillips, Kosobud, \& Belknap, 1990; DeFries, 1981; Henderson, 1989; Hewitt, 1992) as has the use of unselected control lines (Brush, 1992; Crabbe et al., 1990; DeFries, 1981; but see Hill, 1972a, 1972b), recombinant inbred strains (Blizard, 1992; Hegmann \& Possidente, 1981; Hewitt, 1992), and perpetual restarting (Sinclair, 1992). Some authors have recognized the utility of hybrid correlational analysis, or more simply crossing (Blizard, 1992; DeFries, 1981; Hewitt, 1992; Wahlsten, 1992), but usually discuss it as a last recourse if none of the other methods are possible. We agree that the above methods can be useful in some situations; however, when the purpose of a study is to determine the nature of trait correlations, hybrid correlational analysis provides two distinct advantages.

First, it is less resource intensive than virtually all other alternatives. In a divergent selection situation, only high and low lines need to be maintained, which can result in substantial savings in time, effort, and resources. To test whether an unselected trait is genetically correlated with the selected trait, one needs only to test individuals from both parental strains on both measures and then cross the strains reciprocally to produce $F_{1}$ and 
subsequently $\mathrm{F}_{2}$ generation individuals. Then $\mathrm{F}_{2}$ generation individuals are tested on the two traits. A nonzero correlation between the two traits in the $\mathrm{F}_{2}$ generation indicates that there is a genetic basis for the trait correlation seen in the parental lines.

Second, the method of hybrid correlational analysis is not affected by genetic differences that can arise in replicate lines. Gene correlates of behaviors are likely to be degenerate, as is the genetic code. In the genetic code, more than one codon triplet specifies a particular amino acid (Lewin, 1994). Similarly, more than one genotype can be associated with the same behavioral phenotype. It is therefore not unreasonable to assume that replicate or perpetually restarted selection lines may be composed of different genotype constellations that are indistinguishable phenotypically. If this is the case, trait correlations observed in one line with a particular genotype constellation may not be seen in a replicate line with a potentially different genotype constellation. The method of hybrid correlational analysis can be used to reveal the nature of trait correlations in particular selected or inbred lines regardless of their idiosyncratic genetic constellations.

In this study we first report an allozyme survey of the high- and low-geotaxis lines in which we identify genotype-genotype and genotype-phenotype correlations. Then we use $\mathrm{F}_{2}$ correlational analysis and backcrosses to elucidate these correlations as well as a phenotypephenotype correlation previously reported by Lofdahl, Hu, Ehrman, Hirsch, and Skoog (1992).

\section{General Method}

\section{Subjects}

Individuals were taken from the lines of Drosophila melanogaster that intermittently have been divergently selected for geotactic performance and have evolved stable, extreme performance (see Ricker \& Hirsch, 1985, for the selection history of the lines). All flies were raised in an environmental chamber at $25^{\circ} \mathrm{C}, 50 \%$ relative humidity, and a 16:8-hr lightdark cycle with lights on at $0800 \mathrm{hr}$. Flies were cultured in $10.0 \times 3.5 \mathrm{~cm}$ diameter plastic vials with yeasted Instant Drosophila Medium (both supplied by Carolina Biological Supply, Burlington, North Carolina). Maintenance of the high- and low-geotaxis lines consisted of mass transfer of the populations to new food vials every 2-4 weeks. Hence generations overlap. This topic was discussed by Ricker (1984) and is mentioned here only to point out that the generation numbers given are not meant to be exact; in fact, they are surely underestimates of the actual number of generations since the isolation of the two lines.

\section{Geotaxis Testing}

All geotaxis testing took place in multiple unit classification mazes that consisted of a series of 15 choice points where individual flies could walk either up (geo-negative) or down (geo-positive; for a description, see Hirsch, 1959). Sixteen collection tubes were located at the end of the maze where flies that made 15 up choices entered the uppermost tube (15) and those that made 15 down choices entered the lowermost tube (0). All testing was begun before $1200 \mathrm{hr}$ and took place in constant light. Males and females were run separately and were given approximately $24 \mathrm{hr}$ to traverse the maze. 


\section{Experiment 1}

We conducted this allozyme survey to identify potential genotype-phenotype correlations in the selected lines. Correlations between allozyme alleles and geotaxis could be the result of selecting for geotaxis, of genetic drift, or of linkage.

\section{Method}

\section{Sample preparation}

For this survey adult flies were taken from each selected geotaxis line without regard to age or virginity (females) at Generations 707, 724, 729, and 747. Flies were then stored at $-70^{\circ} \mathrm{C}$ until electrophoresed. Individuals were homogenized in 12-17 $\mu$ l of grinding buffer (50 ml tris-citrate/borate gel buffer [Berlocher, 1980] with 6 drops of 2-mercaptoethanol) when electrophoresed in a starch gel. Individuals electrophoresed in acrylamide gels were homogenized in a buffer with tracking dye ( $10.0 \mathrm{~g}$ sucrose, $0.5 \mathrm{mg}$ bromophenol blue, $0.23 \mathrm{~g}$ tris, $0.03 \mathrm{~g}$ citrate, 6 drops 2-mercaptoethanol, up to $50 \mathrm{ml}$ of water, added to $50 \mathrm{ml}$ grinding buffer).

\section{Electrophoresis}

For starch gel electrophoresis each sample was then absorbed by two $5 \times 7 \mathrm{~mm}$ pieces of Whatman (Maidstone, United Kingdom) No. 1 filter paper, and each piece was inserted into a separate $12 \%$ starch gel. Each of the starch gels was cut into three slices that were then stained for different enzymes, so every individual was assayed for at least three different enzyme systems. The enzyme-staining procedures are similar to those described by Murphy, Sites, Buth, and Haufler (1990). Table 1 lists the enzymes surveyed, abbreviations, Enzyme Commission numbers, and the buffer system used for each.

Amylase $(A m y)$ and alcohol dehydrogenase $(A d h)$ were electrophoresed on a vertical polyacrylamide gel in a cold room for $6 \mathrm{hr}$ (see Stoltenberg, 1992, for a description of methods). The electrode buffer (IV, $6.0 \mathrm{~g}$ tris, $28.8 \mathrm{~g}$ glycine per liter of water) was diluted 1:3 for use. First, the gels were soaked in the Adh stain until bands appeared, and then they were soaked in a 2\% starch solution for $1 \mathrm{hr}$ and stained for Amy with Gram's iodine (30.0 g potassium iodide, $13.0 \mathrm{~g}$ iodine, $1 \mathrm{~L}$ water).

Totals of 300 and 299 individuals from the low- and high-geotaxis lines, respectively, were electrophoresed with a minimum of 24 from each line assayed for each allozyme. 
Table 1. Allozymes Surveyed and Their Buffer Systems

\begin{tabular}{llcc}
\hline Allozymes & Abbreviation & \\
\hline Acid phosphatase & Acph-1 & EC number & Buffer system $^{\mathrm{c}}$ \\
Aconitase & Acon & 3.1 .3 .2 & I \\
Alcohol dehydrogenase & Adh & 4.2 .1 .3 & I \\
Aldehyde oxidase & Aldox 1 & 1.1 .1 .1 & I, IV \\
Glycerophosphate dehydrogenase & Gpdh & 1.2 .1 .3 & I \\
Leucine amino peptidase & Lap-A & 1.1 .1 .8 & I \\
Leucine amino peptidase & Lap-D & 3.4 .1 .1 & I \\
Malate dehydrogenase & Mdh-1 [cytoplasmic] & 3.4 .1 .1 & I \\
Malate dehydrogenase & Mdh-2 [mitochondrial] & 1.1 .1 .37 & I \\
Malic enzyme & Men & 1.1 .1 .37 & I \\
Octanol dehydrogenase & Odh & 1.1 .1 .40 & I \\
Phosphoglucomutase & Pgm & 2.7 .5 .1 & I \\
Aldolase & Ald & 4.1 .2 .13 & I \\
Esterase & Est-C & 3.1 .1 .1 & II \\
Esterase & Est-6 & 3.1 .1 .1 & II \\
Fumarase & Fum & 4.2 .1 .2 & II \\
Glutamate oxaloacetic transaminase & Got-1 & 2.6 .1 .1 & II \\
Glutamate oxaloacetic transaminase & Got-2 & 2.6 .1 .1 & II \\
Xanthine dehydrogenase & Xdh [ry] & 1.2 .1 .37 & II \\
Glucose-6-phosphate dehydrogenase & Zw & 1.1 .1 .49 & II \\
6-phosphogluconate dehydrogenase & Pgd & 1.1 .1 .44 & III \\
Amylase & Amy & 3.2 .1 .1 & III \\
\hline
\end{tabular}

aLindsley \& Zimm (1992). 'bnzyme Commission numbers. cBuffer Systems I, II, and III are described by Murphy, Sites, Buth, and Haufler (1990; Tris-citrate/borate, Tris-citrate II, and Tris-borate-EDTA II, respectively). The gel buffer in System I is described by Berlocher (1980).

\section{Results and Discussion}

Three of the 22 allozymes assayed (Adh, Amy, and 6-phosphogluconate dehydrogenase $[P g d]$ ) were found to be fixed for alternative alleles (we use allele instead of electromorph for brevity) in the selected lines. Richmond (1984) and McKechnie (1988) have previously found fixation for the alternative alleles of $A d h, A d h^{\mathrm{s}}$ (slow) in the high-geotaxis line and $A d h^{\mathrm{F}}$ (fast) in the low-geotaxis line. We found the remaining 19 allozyme loci to be fixed for the same allele in both the high- and low-geotaxis lines. That is, no electrophoretic variation was detected between lines at 19 loci that are polymorphic to varying degrees in $D$. melanogaster (see Band, 1975; Berger, 1971; Cabrera, Gonzalez, Larruga, \& Gullon, 1982; Gonzalez, Cabrera, Larruga, \& Gullon, 1982; Kojima, Gillespie, \& Tobari, 1970; Singh, Hickey, \& David, 1982). No allozyme variation was detected within either the high- or low-geotaxis lines at the 22 loci surveyed.

The low-geotaxis line is fixed for the $A m y^{1}$ allele, which is characterized by a single rather fast migrating band. That the Amy allele found in the low-geotaxis selected line is $A m y^{1}$ was confirmed by running individuals from a known pure breeding $A m y^{1}$ line alongside low-geotaxis line individuals on the same gel. The high-geotaxis line Amy allele appears to be $A m y^{2,3}$, but this could not be confirmed by the best direct comparison. However, 
when high-geotaxis line individuals were run side by side in gels with individuals from a pure breeding Amy line and others from a pure breeding $A m y^{3,6}$ line, the slowest migrating band of the high-geotaxis line is directly adjacent to the 3 band of the $A m y^{3,6}$ line, which indicates that it is a 3 band. The faster migrating high-geotaxis line Amy band lies at a position intermediate between the 3 band of the $A m y^{3,6}$ line and the single band of the $A m y^{1}$ line, which indicates that the intermediate band is a 2 . F $F_{1}$ hybrids of the high- and lowgeotaxis lines exhibit three bands that are spaced approximately equidistant from one another, as would be expected of an $A m y^{1 / 2,3}$ heterozygote. Thus, it appears that the allele fixed in the high-geotaxis line is $A m y^{2,3}$.

The third locus fixed for alternative alleles between the lines is $P g d$. We were unable to obtain reference lines homozygous for known alleles of $P g d$, and therefore the allele designations have not been made. The low-geotaxis line, however, has a slower migrating allele, and the high-geotaxis line a faster migrating allele; whereas only two spontaneously occurring alleles of Pgd have been observed in D. melanogaster (Lindsley \& Zimm, 1992), it is a reasonable inference that the alleles found in the high- and low-geotaxis lines are those called A and B, respectively.

In this allozyme survey we found that $A d h, A m y$, and $P g d$ are fixed for alternative alleles in the high- and low-geotaxis lines. The remaining 19 loci surveyed are fixed for the same allele in both lines. No allozyme variation was detected within either selected line at any of the 22 loci examined. The allozyme loci included in this survey are distributed on each of the three major chromosomes with at least two loci on each chromosome arm (Figure 1).
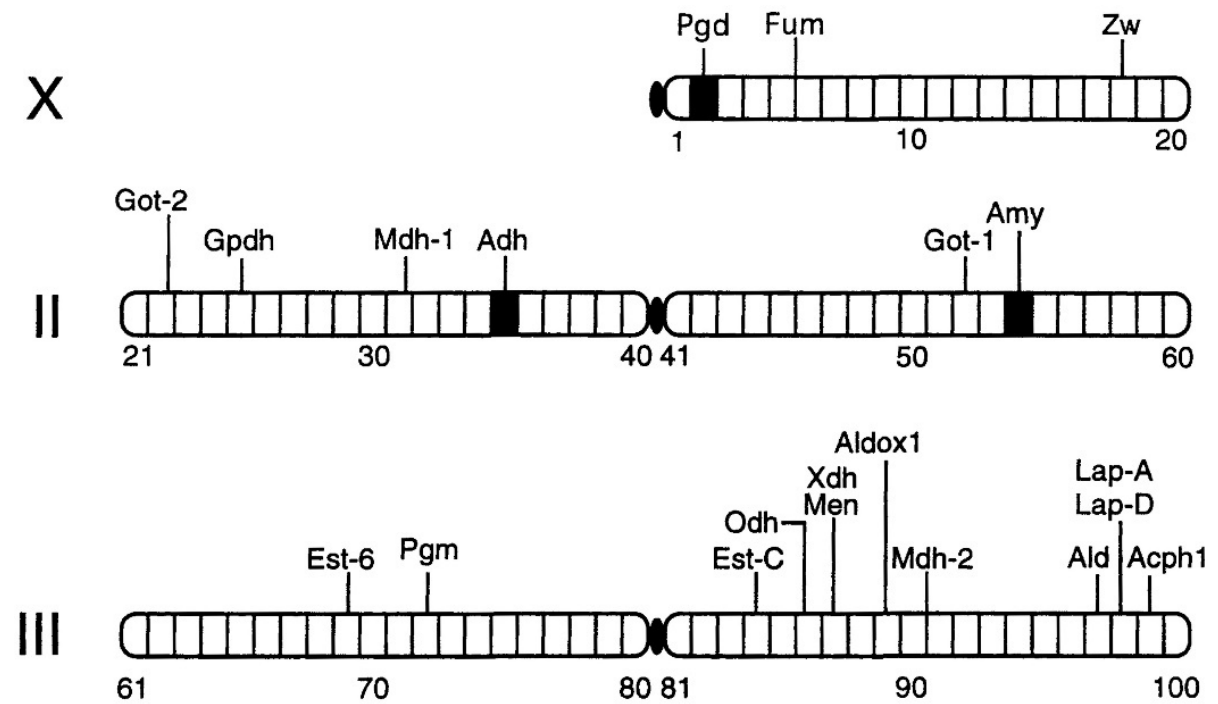

Figure 1. Schematic representation of the cytological map positions of the structural genes for the allozymes surveyed (map positions from Lindsley \& Zimm, 1992). Darkened bands indicate allozymes that are fixed for alternative alleles in the high- and low-geotaxis lines, and darkened ovals represent centromeres. The remaining 18 allozymes (aconitase is unmapped) are fixed for the same allele in the two lines. (Table 1 gives the abbreviations for the allozymes.) 
Alleles of Adh, Amy, and Pgd are correlated in the selected lines with each other (genotype-genotype) and with geotaxis (genotype-phenotype). It is possible that these correlations are the direct result of selection for geotaxis. However, it is more likely that the correlations are the result of genetic drift, given the histories of the lines (see Ricker \& Hirsch, 1985, 1988b). These correlations were assessed in backcrosses and in $F_{2}$ correlational analyses.

\section{Experiment 2}

This experiment assessed the linkage relation (genotype-genotype correlation) between $\mathrm{ADH}$ and AMY on Chromosome II in the high- and low-geotaxis lines. Any recombination supression between ADH and AMY would most likely be detected with these methods.

\section{Method}

\section{Subjects}

The individuals used as parents in the initial reciprocal crosses were taken from the highand low-geotaxis lines at selected Generation 713 and were maintained as described in the General Method. Ten single pair matings (i.e., high-geotaxis [H] female $\times$ low-geotaxis [L] male and $\mathrm{L}$ female $\times \mathrm{H}$ male) were made for each reciprocal cross in $7 \times 2 \mathrm{~cm}$ glass vials with medium. The vials were kept in the environmental chamber.

\section{Husbandry}

Three to 4 days after the initial crosses were made, each set of parents was transferred to fresh food vials. Thirteen to 14 days after the initial crosses, progeny to be used as parents for the next generation were lightly etherized, within $4.5 \mathrm{hr}$ of eclosion, and males were discarded. Ten single pair backcrosses were made by placing an $\mathrm{F}_{1}$ female into a fresh $7 \times$ $2 \mathrm{~cm}$ diameter glass vial with a male from the appropriate selected line (i.e., HL female $\times$ $\mathrm{H}$ male, HL female $\times \mathrm{L}$ male, $\mathrm{LH}$ female $\times \mathrm{H}$ male, and LH female $\times \mathrm{L}$ male). As with the previous generation, parents were transferred to fresh food vials $3-4$ days after the cross. Eleven to 12 days after the backcrosses, progeny began to eclose and were collected under ether anesthesia. Male and female progeny from the same family were stored together in $10.0 \times 3.5 \mathrm{~cm}$ plastic vials with medium until approximately 200 individuals were collected. For each of the four backcross types, four to six separate families were maintained. A family consisted of backcross offspring that resulted from a single pair mating of an $\mathrm{F}_{1}$ female (HL or LH) to a selected line male (H or L). When approximately 200 individuals from a given family were collected, they were stored at $-70^{\circ} \mathrm{C}$ until electrophoresis.

\section{Electrophoresis}

Polyacrylamide gel electrophoresis was carried out as described for Experiment 1. Gels were stained for both $A d h$ and Amy. At least 55 individuals were electrophoresed for each backcross type from three families per type. A total of 264 backcross individuals was assayed. 


\section{Results and Discussion}

Genes ADH and AMY are located on Chromosome II at map locations 50.1 and 77.7, respectively, which results in an expected recombination rate of 0.212 . Table 2 presents the frequency of observed genotypes for each backcross type. The observed recombination rate, $0.227\left(\sigma^{2}=0.0006\right)$, was calculated based on the known map distance between two loci (Weir, 1990; presented originally by Haldane, 1919). The expected and observed recombination rates were compared by use of the arcsine transformation given in Cohen (1988) and were not found to be significantly different $\left(h_{\text {obtained }}=0.048, h_{\text {critical }}=0.230, \alpha=0.01\right.$, twotailed, with $N=250$ ). Therefore, there is no evidence for recombination suppression between ADH and AMY genes in the lines. The genotype-genotype correlation observed in the high- and low-geotaxis lines was not likely to be caused by a genetic correlation between Adh and Amy.

Table 2. Number of Progeny Resulting from Each Backcross Type

\begin{tabular}{|c|c|c|c|c|}
\hline \multirow[b]{2}{*}{ Genotype (Adh-Amy) } & \multicolumn{4}{|c|}{ Backcross type } \\
\hline & $\mathrm{HL}+\times \mathrm{L}{ }^{\lambda}$ & $\mathrm{LH}+\times \mathrm{L}^{\pi}$ & $\mathrm{HL}+\times \mathrm{H}^{\lambda}$ & $\mathrm{LH} q \times \mathrm{H} \partial$ \\
\hline$\underline{S-2,3}$ & 20 & 27 & & \\
\hline \multicolumn{5}{|l|}{$\overline{F-1}$} \\
\hline$\underline{S-1}$ & 8 & 7 & & \\
\hline \multicolumn{5}{|l|}{$\overline{\mathrm{F}-1^{\mathrm{a}}}$} \\
\hline$\underline{F-2,3}$ & 4 & 10 & & \\
\hline \multicolumn{5}{|l|}{$\mathrm{F}-1^{\mathrm{a}}$} \\
\hline$\underline{F-1}$ & 23 & 26 & & \\
\hline \multicolumn{5}{|l|}{$\overline{F-1}$} \\
\hline$\underline{S-2,3}$ & & & 21 & 30 \\
\hline \multicolumn{5}{|l|}{$\overline{S-2,3}$} \\
\hline$\underline{S-1}$ & & & 4 & 5 \\
\hline \multicolumn{5}{|l|}{$S-2,3^{a}$} \\
\hline$\underline{F-2,3}$ & & & 9 & 13 \\
\hline \multicolumn{5}{|l|}{$S-2,3$} \\
\hline$\underline{F-1}$ & & & 26 & 31 \\
\hline$S-2,3$ & & & & \\
\hline
\end{tabular}

Note: Rows indicate genotype, columns indicate backcross type $\left(\mathrm{F}_{1}+\times\right.$ parental line $\left.\widehat{\delta}\right) . \mathrm{S}$ and $\mathrm{F}$ describe relative migration rates of alternative alleles. $\mathrm{H}=$ high geotaxis; $\mathrm{L}=$ low geotaxis; $\mathrm{S}=$ slow; $\mathrm{F}=$ fast.

aRecombinant types.

\section{Experiment 3}

In this experiment we performed a hybrid correlational analysis to assess the association of both Adh and Amy with geotactic performance (genotype-phenotype correlation) in the selected high- and low-geotaxis lines. That $P g d$ is also fixed for alternative alleles in the selected lines was discovered after the initiation of this experiment. Therefore, the relation between $P g d$ and geotaxis remains yet to be examined. 


\section{Method}

Subjects

At Generations 692 and 699, adults were cleared from their vials in both lines and within 5-6 hr (to assume virginity) 50 newly eclosed individuals of each sex per line were collected under ether anesthesia. We then placed five high-geotaxis females with five low-geotaxis males in each of 10 vials to found the HL subline, and the reciprocal combination (lowgeotaxis females $\times$ high-geotaxis males) to found the LH subline. To breed the resulting $\mathrm{F}_{1}$ generation, the same procedures were followed except no reciprocal crosses were made; that is, matings were limited to those within subline (i.e., HL females $\times$ HL males and LH females $\times$ LH males). The HL and LH sublines were cultured and tested separately throughout.

From each subline, within 3-4 hr of eclosion, samples of approximately 230 males and 230 females were collected and maintained sexually segregated in vials of approximately 115 flies for 2-3 days in the environmental chamber used for geotaxis testing.

One sample of approximately $200 \mathrm{~F}_{2}$ individuals of each sex from each subline (HL and LH) collected from vials used to generate subjects for Experiment 4 was tested for geotactic performance, stored at $-70^{\circ} \mathrm{C}$, and then electrophoresed (as described for Experiment 1 ).

\section{Statistics}

Each fly was tested for geotactic performance, Adh, and Amy. Observed allozyme zygosity ratios at each locus were compared to Mendelian expectations (i.e., 1:2:1) using the chisquare goodness-of-fit test. Analysis of variance (ANOVA) was used to examine the effects of subline, sex, Adh, Amy, and associated interactions on geotactic performance. Sums of squares were calculated with the partial least squares method (Type III ANOVA).

\section{Results and Discussion}

Results of the electrophoretic assay indicate that the observed zygosity ratios in the overall $\mathrm{F}_{2}$ generation sample did not differ significantly from 1:2:1 Mendelian expectations for $A d h$, $\chi^{2}(2, N=773)=2.26, .25<p<.5$, or $A m y, \chi^{2}(2, N=773)=0.62, .5<p<.75$. Table 3 gives the observed (and expected) frequencies of homozygotes and heterozygotes for both allozyme loci for the entire $\mathrm{F}_{2}$ generation sample as well as the categories defined by subline and sex (e.g., HL females). Chi-square statistics and sample sizes are also given. It is interesting to note that Amy in the LH male sample did not conform to Mendelian expectations, $\chi^{2}(2$, $N=173)=12.86, p<.005$. This could indicate that (a) a biased sample of LH males was assayed or (b) Amy zygosity is related to fitness in some way in males from the LH sample. 
Table 3. Frequencies of Adh and Amy Genotypes Observed (and Expected) in the F2 Generation

\begin{tabular}{|c|c|c|c|c|c|}
\hline & Pooled $F_{2}$ & $\mathrm{HL} \propto q$ & HL ठへ & $\mathrm{LH}+9$ & LH ठへ \\
\hline \multicolumn{6}{|c|}{ Adh } \\
\hline $\mathrm{F} / \mathrm{F}$ & $211(193.25)$ & $61(54.0)$ & $60(51.25)$ & $54(44.75)$ & $36(43.25)$ \\
\hline $\mathrm{F} / \mathrm{S}$ & $378(386.5)$ & $103(108.0)$ & $90(102.5)$ & $81(89.5)$ & $104(86.5)$ \\
\hline $\mathrm{S} / \mathrm{S}$ & $184(193.25)$ & $52(54.0)$ & $55(51.25)$ & $44(44.75)$ & $33(43.25)$ \\
\hline $\mathrm{N}$ & 773 & 216 & 205 & 179 & 173 \\
\hline$\chi^{2}$ & 2.26 & 1.21 & 3.29 & 2.73 & 7.18 \\
\hline \multicolumn{6}{|c|}{ Amy } \\
\hline $1 / 1$ & $186(193.25)$ & $61(54.0)$ & $51(51.25)$ & $47(44.75)$ & $27(43.25)$ \\
\hline $1 / 2,3$ & $397(386.5)$ & $104(108.0)$ & $101(102.5)$ & $83(89.5)$ & $109(86.5)$ \\
\hline $2,3 / 2,3$ & $190(193.25)$ & $51(54.0)$ & $53(51.25)$ & $49(44.75)$ & $37(43.25)$ \\
\hline $\mathrm{N}$ & 773 & 216 & 205 & 179 & 173 \\
\hline$\chi^{2}$ & 0.62 & 1.22 & 0.08 & 0.99 & $12.86^{*}$ \\
\hline
\end{tabular}

Note: Expected values were obtained under the hypothesis of a 1:2:1 ratio of genotypes in the $\mathrm{F}_{2}$ generation and are shown in parentheses. $\mathrm{H}=$ high geotaxis; $\mathrm{L}=$ low geotaxis. ${ }^{*} p<.005$.

The distribution of geotaxis scores for the pooled $F_{2}$ sample is shown in Figure 2. The pile-ups seen in Categories 0 and 15 may have been due to the mazes' restricted range of measurement (which keeps all flies that would have continued up or down from doing so) or because the limited recombination, which is expected to occur by the $\mathrm{F}_{2}$ generation (given that male recombination is rare in D. melanogaster), ought to produce flies with large blocks of genes from the selected lines.

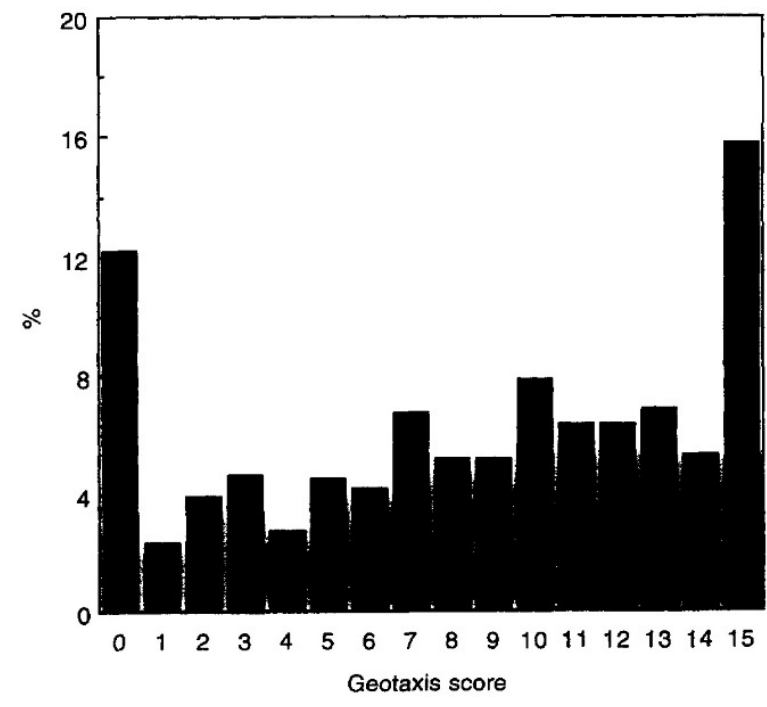

Figure 2. Percentage of $\mathrm{F}_{2}$ generation individuals with a given geotaxis score pooled over subline (HL and $\mathrm{LH})$ and $\operatorname{sex}(N=776) . \mathrm{H}=$ high geotaxis; $\mathrm{L}=$ low geotaxis.

Eleven interaction terms are included in the model tested (see Table 4); however, because male recombination is rare and ADH and AMY genes are located on the same chromosome, certain cells in the matrix occurred at low frequencies, which makes the interpretation of 
interactions difficult. This, coupled with the low power to detect interactions with this statistical procedure (Wahlsten, 1990), leads us to focus our attention on the main effects. Inclusion of the interaction terms in the model, however, enables us to account for the variance due to the interactions and thereby obtain a clearer picture of variance due to the main effects.

Table 4. Partitioning of Variance of Geotaxis Scores of $\mathrm{F}_{2}$ Generation from Reciprocal Hybridization of the High and Low Lines $(N=773)$

\begin{tabular}{lrrl}
\hline Source & $d f$ & $F$ & $p$ \\
\hline Model & 27 & 8.63 & .0001 \\
Subline & 1 & 2.90 & .09 \\
Sex & 1 & 37.91 & .0001 \\
Amy & 2 & 0.57 & .56 \\
Adh & 2 & 12.89 & .0001 \\
Subline $\times$ Sex & 1 & 6.07 & .01 \\
Subline $\times$ Amy & 2 & 2.89 & .06 \\
Subline $\times$ Adh & 2 & 0.46 & .63 \\
Sex $\times$ Amy & 2 & 1.78 & .17 \\
Sex $\times$ Adh & 2 & 0.34 & .71 \\
Amy $\times$ Adh & 2 & 1.25 & .29 \\
Subline $\times$ Sex $\times$ Amy & 2 & 0.32 & .73 \\
Subline $\times$ Sex $\times$ Adh & 2 & 1.00 & .37 \\
Subline $\times$ Amy $\times A d h$ & 2 & 1.12 & .33 \\
Sex $\times A m y \times A d h$ & 2 & 1.89 & .15 \\
Subline $\times$ Sex $\times A m y \times A d h$ & 2 & 4.63 & .01 \\
\hline
\end{tabular}

Table 4 shows that sex $(p=.0001)$ and $\operatorname{Adh}(p=.0001)$ explain a significant amount of variance in geotactic performance of $\mathrm{F}_{2}$ generation hybrids, whereas subline $(p=.09)$ and Amy $(p=.56)$ do not. The finding of a sex effect on geotactic performance is consistent with the chromosome analyses performed on the selected lines (Erlenmeyer-Kimling, Hirsch, \& Weiss, 1962; Hirsch \& Ksander, 1969; Ricker \& Hirsch, 1988a, 1988b), all of which found significant $X$ chromosome effects. $Y$ chromosome effects may also be reflected in the sex effect. However, Ricker (1984) did not find them to be significant in the $\mathrm{F}_{2}$ generation (but see Stoltenberg \& Hirsch, 1994). Sex accounted for $3.77 \%$ of the variation observed in geotactic performance.

Figures 3 and 4 present the relations between Adh, Amy, and geotaxis. Figure 3 shows that, within each Adh genotype (presented on the abscissa), substitution of the alternative Amy allele has no effect on mean geotaxis scores. Figure 4, on the other hand, shows that, within each Amy genotype, substituting the alternative Adh allele has statistically significant effects on mean geotaxis score. An exception can be seen in the Amy heterozygote where the mean geotaxis score of the $A d h^{\mathrm{F}}$ homozygotes is not statistically different from that of the $A d h$ heterozygote. The large error bars seen in this case indicate the rather small sample of Adh homozygote/Amy heterozygote flies tested for geotactic performance $(N=51)$. The substitution of $A d h^{\mathrm{s}}$ results in significantly different geotaxis means only in Amy homozygotes, not in Amy heterozygotes. 


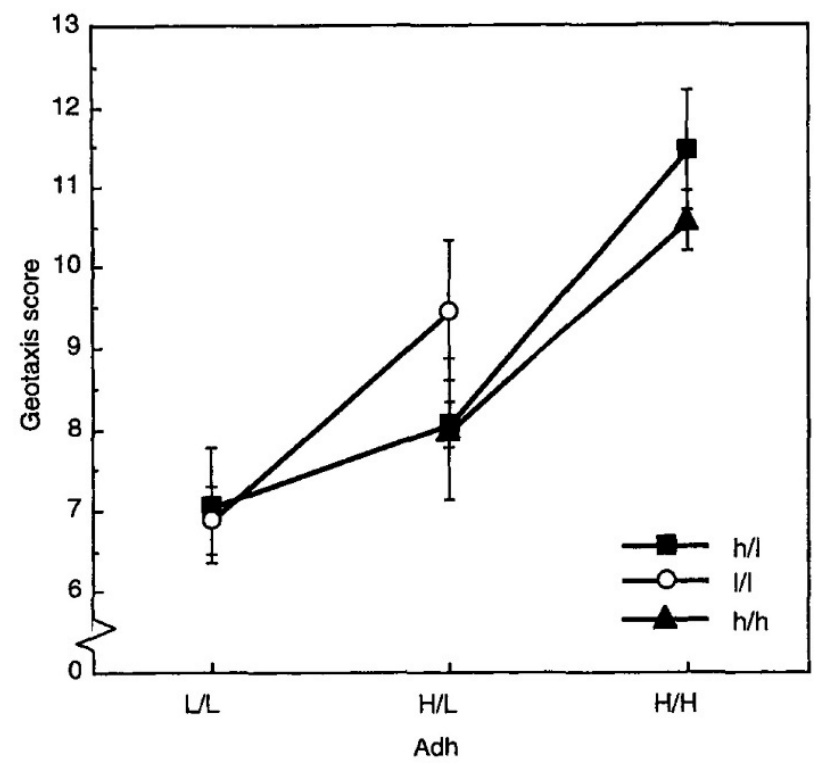

Figure 3. Mean geotaxis scores (with standard error bars) of the $\mathrm{F}_{2}$ generation with specific alcohol dehydrogenase $(A d h)$ and amylase $(A m y)$ genotypes $\left(A d h^{\mathrm{F}}=\mathrm{L}, A d h^{\mathrm{S}}=\mathrm{H}, A m y^{1}=1\right.$, and $\left.A m y^{2,3}=\mathrm{h}\right)$. Within each $A d h$ genotype, alternative $A m y$ genotypes do not have significantly different geotaxis means.

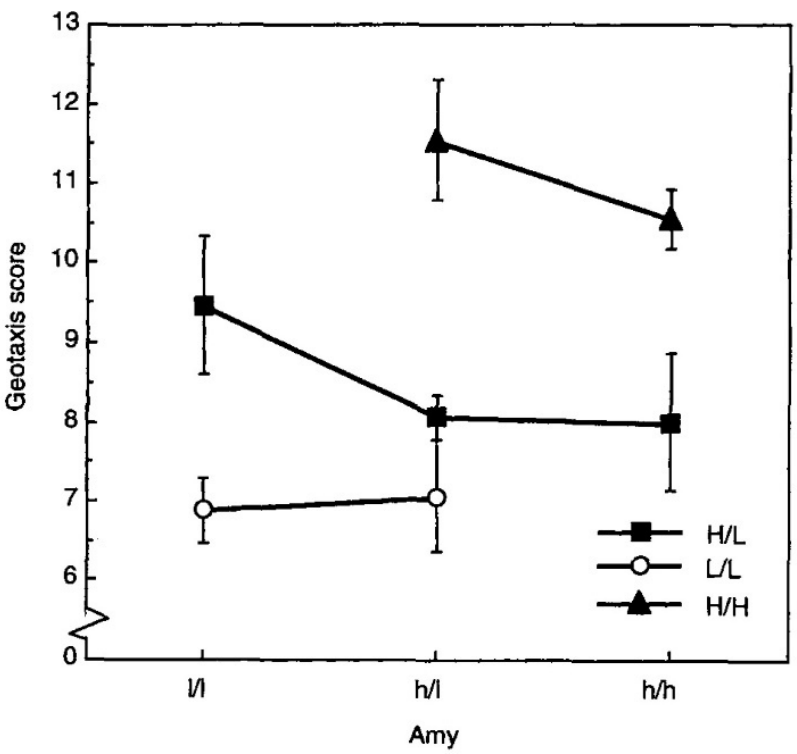

Figure 4. Mean geotaxis scores (with standard error bars) of the $\mathrm{F}_{2}$ generation with specific amylase $(A m y)$ and alcohol dehydrogenase $(A d h)$ genotypes $\left(A m y^{1}=1, A m y^{2,3}=\mathrm{h}, A d h^{\mathrm{F}}=\right.$ $\mathrm{L}$, and $\left.A d h^{\mathrm{S}}=\mathrm{H}\right)$. Within each $A m y$ genotype, alternative genotypes $A d h$ have significantly different geotaxis means, except for one case in Amy heterozygotes. 
Adh accounted for $2.43 \%$ of the variation observed in geotactic performance. This suggests that on Chromosome II in the region around the ADH gene (50.1) there is a gene involved in the genetic systems that influence geotaxis in the high- and low-geotaxis lines. The correlation between Adh and geotaxis, observed in the selected lines, appears to be a genotype-phenotype correlation, whereas the correlation between Amy and geotaxis may be genetic drift.

\section{Experiment 4}

This experiment presents an $\mathrm{F}_{2}$ correlational analysis of high- and low-geotaxis lines to examine the phenotype-phenotype correlation between geotactic performance and mate preference reported by Lofdahl et al. (1992). In a multiple-choice mating situation, approximately $60 \%$ of matings were homogamic, which indicates partial premating reproductive isolation between the selected lines.

\section{Method}

Subjects

Flies were prepared and tested for geotaxis as described in Experiment 3. After same-sex geotaxis testing, from each of seven samples per sex, the 26 most extreme geo-positive and the 26 most extreme geo-negative flies were tested for mate preference. If the most extreme collection tubes (i.e., 15 and 0) did not contain enough flies, the next most extreme tubes were used until a sufficient number of flies was collected. In most cases, only two or three other tubes were needed to obtain 26 flies; however, in one case eight tubes were used.

Both males and females from one geotactic extreme (high or low) were briefly etherized, and the distal end of one wing was clipped for identification. The wing clipping was counterbalanced by geotactic extreme and by wing (right vs. left).

\section{Mate preference testing}

We used the method of Elens and Wattiaux (1964) to assay mate preference as in Lofdahl et al. (1992). We aspirated into the chamber 5- to 7-day-old flies, first males and then females, 11 of a sex from each geotactic extreme for a total of 44 . Each chamber was observed for $1 \mathrm{hr}$ by two observers who recorded the location of each mating, whether the participants had clipped or unclipped wings, and the 6-min interval in which the mating occurred. Observations were begun before $1200 \mathrm{hr}$ and took place under a fluorescent lamp.

\section{Results and Discussion}

Of the 56 samples of $\mathrm{F}_{2}$ generation individuals assayed for mate preference, 51 had mean geotaxis scores that were as extreme or more extreme than those of the corresponding selected line. Therefore, the geotaxis scores of the majority of $\mathrm{F}_{2}$ generation individuals assayed for mate preference were indistinguishable from those of selected line flies. Figure 5 compares the mean geotaxis scores of the $\mathrm{HL}$ and $\mathrm{LH} \mathrm{F}_{2}$ generation males and females assayed for mate preference with mean scores from the corresponding geotaxis line. 


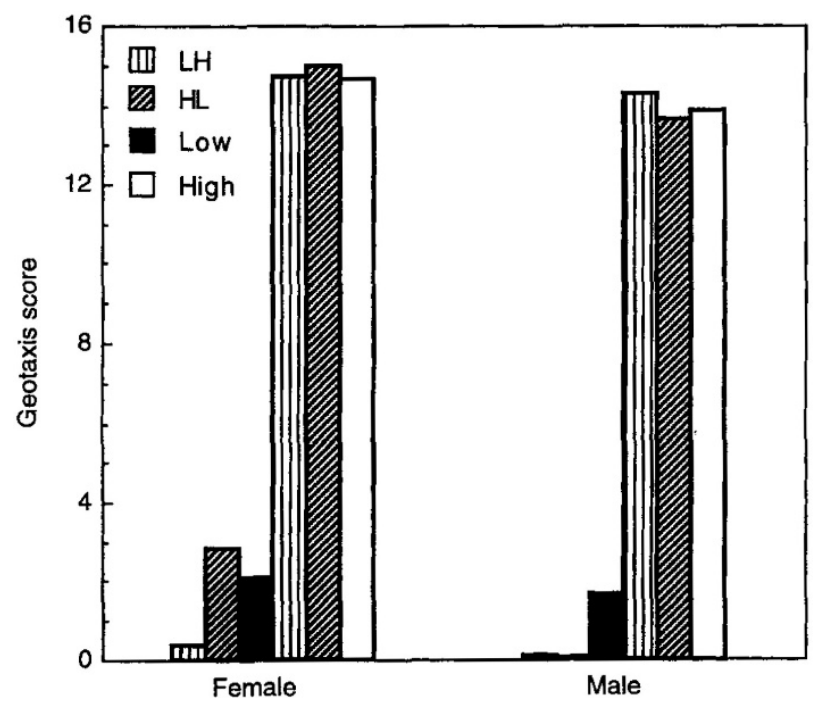

Figure 5. Mean geotaxis scores of the extreme-scoring $\mathrm{F}_{2}$ generation flies (both low and high) tested for mate preference and of samples of the corresponding low- and high-geotaxis lines (at Generation 703) for comparison. LH and HL indicate hybrid sublines tested separately throughout. The sample size in each case was approximately 200.

The results of the mate preference assay indicate that $\mathrm{F}_{2}$ generation flies, which are hybrids of the high- and low-geotaxis lines, mate without respect to geotactic extreme performance. Table 5 presents the frequency of occurrence of each type of mating observed. In each case ( $\mathrm{LH}, \mathrm{HL}$, and when samples are pooled), the chi-square test for association was less than one. Thus, mate preference and geotactic extreme behavior, which are associated in the high- and low-geotaxis lines, are not associated in their $\mathrm{F}_{2}$ hybrids. The mate preference assay is large enough $(N=560)$ to detect the difference between $50 \%$ (i.e., random mating) and $60 \%$ homogamy (Lofdahl et al., 1992) with $96 \%$ power at an alpha of .05 (Cohen, 1988).

\begin{tabular}{lcc}
\hline \multicolumn{2}{l}{ Table 5. Mating Frequencies of $\mathrm{F}_{2}$ Generation Individuals Classified Geotactically } \\
\hline Female & Male & $n$ \\
\hline High & High & 145 \\
Low & Low & 136 \\
High & Low & 137 \\
Low & High & 142 \\
Total & & 560 \\
\hline
\end{tabular}

Note: No association between geotaxis extreme behavior and mate preference in $\mathrm{F}_{2}$ generation, test for association, $\chi 2(1)=0.0007, p>.90$

These results show that the phenotype-phenotype correlation between geotactic performance and mate preference in the high- and low-geotaxis lines is not maintained after hybridization. Therefore, the phenotype-phenotype correlation between the two behavior 
traits in the selected lines is not the pleiotropic effect of a single genetic system, nor is it due to correlated genetic systems.

\section{General Discussion}

We draw three conclusions from this study. First, allelic variation in Adh is genetically correlated with variation in geotactic performance in the high- and low-geotaxis lines. This result brings us a step closer to finding gene correlates of geotaxis because the Adh is a gene product (allozyme) correlate. With the exception of two studies, the most refined level of analysis in the search for genetic correlates of geotaxis in D. melanogaster to date has been the chromosome (Hirsch \& Erlenmeyer-Kimling, 1962; Hirsch \& Ksander, 1969; Pyle, 1978; Ricker \& Hirsch, 1988a, 1988b). Kessler and Kraemer (1975) demonstrated, in allele substitution experiments with eye color mutants, that the white apricot allele influenced geotactic performance. McMillan and McGuire (1992) recently reported that male flies homozygous for the homeotic mutant gene spineless-aristapedia exhibited extreme positive geotactic performance that was not due solely to the presence of ectopic leg tissue. These appear to be the only reports of specific gene correlates of geotaxis in D. melanogaster.

The present study is the first to describe an allozyme correlate of geotaxis, Adh; it explains a significant amount of variance in geotactic performance of $\mathrm{F}_{2}$ generation hybrids between the high- and low-geotaxis lines. Thus, it appears that a gene on the second chromosome in the region of the ADH gene (50.1) is involved in the genetic systems that influence geotaxis in the selected lines.

Second, the phenotype-phenotype correlation between geotactic performance and mate preference, observed in the high- and low-geotaxis lines (Lofdahl et al., 1992), is not the result of a shared genetic system (i.e., pleiotropy). The partial premating reproductive isolation demonstrated in the geotaxis lines was not observed in their $\mathrm{F}_{2}$ generation hybrids and is therefore not due to a correlation between the genetic correlates of geotaxis and those of mate preference. Geotactic performance may be considered a component of habitat preference, which has been theorized to facilitate speciation (e.g., Diehl \& Bush, 1989), if genetically correlated with mate preference.

Recently, Rice and Salt (1990) provided empirical evidence that premating reproductive isolation had arisen as a correlated character in response to selection for divergent habitat preference in D. melanogaster. They performed 35 generations of selection based on phototaxis, geotaxis, chemotaxis, and developmental time in complex mazes constructed to simulate natural conditions. As the experiment progressed, increasing habitat specialization resulted in increased reproductive isolation between the populations. The flies apparently traversed the mazes and arrived at their preferred habitats before reaching sexual maturity, and thereby they reduced the opportunity to mate to only those opposite sex flies that had reached the same habitat. It is interesting to note that when flies with divergent habitat preferences were tested in forced consolidation mating tests, similar to the tests that have shown mate preference in the geotaxis lines, no positive assortative mating occurred (Rice \& Salt, 1990). Thus, it appears that the premating reproductive isolation that has evolved between the high- and low-geotaxis lines is qualitatively different from that observed by Rice and Salt. The correlation between habitat preference and mate preference 
reported by Rice and Salt is solely a byproduct of the spatiotemporal separation of the flies in their preferred habitats. It may be the case that mate and habitat preference need not be genetically correlated to facilitate speciation if the drive to reach divergent habitats is strong (as in the high- and low-geotaxis lines) and mating is postponed until arrival at the preferred habitats.

Third, the four experiments presented in this article provide further evidence of the efficacy of hybrid correlational analysis in determining the nature of trait correlations in isolated, selected or inbred, lines.

The identification of trait correlations in selected or inbred lines is only a first step in characterizing them. Hybrid correlational analysis provides us with a straightforward approach to describe trait correlations that exploits the power of meiosis. In this article we have identified correlations (a) between Adh, Amy, and Pgd and (b) between Adh, Amy, Pgd, and geotaxis, and we have used backcrosses and $\mathrm{F}_{2}$ correlational analysis to characterize the correlations (a) between Adh and Amy (genotype-genotype), (b) between Adh, Amy, and geotaxis (genotype-phenotype), and (c) between geotaxis and mate preference (phenotypephenotype). Only the correlation between $A d h$ and geotaxis survived meiosis, which indicates that a gene in the region surrounding the ADH gene on Chromosome II (50.1) may be involved in geotactic performance. All other correlations we examined were eliminated after hybridization, which suggests that they were the result of genetic drift.

That the majority of the strong correlations observed in the selected lines proved to be chance effects ought to be considered when trait correlations are observed in any species. In situations where breeding analyses are difficult (or impossible), the identification of trait correlations is often the the final stage of the analysis. Our evidence indicates that even strong correlations (e.g., Amy and geotaxis) can result from genetic drift and are therefore likely to be of little importance in understanding heredity-behavior relations.

Further research with these lines can examine (a) the correlation between $P g d$ and geotaxis, (b) the correlations between Pgd, Amy, and Adh, (c) the region of Chromosome II surrounding the $\mathrm{ADH}$ gene for genes associated with geotaxis, and (d) the relations between Adh, Amy, Pgd, and mate preference.

Acknowledgments - This work was supported by funds from the University of Illinois Foundation. We thank Hu Dan and Katharine Lofdahl for their help in making the mate preference observations and for helpful discussions and Sheila Lyons for technical assistance.

\section{References}

Band, H. T. (1975). A survey of isozyme polymorphism in a Drosophila melanogaster natural population. Genetics, 80, 761-771.

Berger, E. M. (1971). A temporal survey of allelic variation in natural and laboratory populations of Drosophila melanogaster. Genetics, 67, 121-136.

Berlocher, S. H. (1980). An electrophoretic key for distinguishing species of the genus Rhagoletis (Diptera: Tephritidae) as larvae, pupae, or adults. Annals of Entomological Society of America, 73, 131-137.

Blizard, D. A. (1992). Analyzing phenotypic correlations in studies with selected lines. Behavior Genetics, 22, 29-33. 
Brush, F. R. (1992). Which twin has the Toni? Commentary on J. D. Sinclair. Behavior Genetics, 22, 25-27.

Cabrera, V. M., Gonzalez, A. M., Larruga, J. M., \& Gullon, A. (1982). Electrophoretic variability in natural populations of Drosophila melanogaster and Drosophila simulans. Genetica, 59, 191-201.

Cohen, J. (1988). Statistical power analysis for the behavioral sciences (2nd ed.). Hillsdale, NJ: Erlbaum.

Crabbe, J. C., Phillips, T. J., Kosobud, A., \& Belknap, J. K. (1990). Estimation of genetic correlation: Interpretation of experiments using selectively bred and inbred animals. Alcoholism: Clinical Experimental Research, 14, 141-151.

DeFries, J. C. (1981). Selective breeding for behavioral and pharmacological responses in laboratory mice. In E. S. Gershon, S. Matthysse, X. O. Breakfield, \& R. D. Ciarnello (Eds.), Genetic research strategies for psychobiology and psychiatry (pp. 199-214). Pacific Grove, CA: Boxwood Press.

Diehl, S. R., \& Bush G. L. (1989). The role of habitat preference in adaptation and speciation. In D. Otte \& J. A. Endler (Eds.), Speciation and its consequences (pp. 345-365). Sunderland, MA: Sinauer Associates.

Elens, A., \& Wattiaux, J. M. (1964). Direct observation of sexual isolation. Drosophila Information Service, 39, 118-119.

Erlenmeyer-Kimling, L., Hirsch, J., \& Weiss, J. M. (1962). Studies in experimental behavior genetics: III. Selection and hybridization analyses of individual differences in the sign of geotaxis. Journal of Comparative and Physiological Psychology, 55, 722-731.

Falconer, D. S. (1989). Introduction to quantitative genetics (3rd ed.). New York: Longman.

Gonzalez, A. M., Cabrera, V. M., Larruga, J. M., \& Gullon, A. (1982). Genetic distance in the sibling species Drosophila melanogaster, Drosophila simulans and Drosophila mauritiana. Evolution, 36, 517-522.

Haldane, J. B. S. (1919). The combination of linkage values and the calculation of distances between loci of linked factors. Journal of Genetics, 8, 299-309.

Hegmann, J. P., \& Possidente, B. (1981). Estimating genetic correlations from inbred strains. Behavior Genetics, 11, 103-114.

Henderson, N. D. (1989). Interpreting studies that compare high- and low-selected lines on new characters. Behavior Genetics, 19, 473-502.

Hewitt, J. K. (1992). Localization of the primary sites of genetic influence requires more information than two selected lines alone can yield. Behavior Genetics, 22, 19-20.

Hill, W. G. (1972a). Estimation of genetic change: I. General theory and design of control populations. Animal Breeding Abstracts, 40, 1-15.

Hill, W. G. (1972b). Estimation of genetic change: II. Experimental evaluation of control populations. Animal Breeding Abstracts, 40, 193-213.

Hirsch, J. (1959). Studies in experimental behavior genetics: II. Individual differences in geotaxis as a function of chromosome variations in synthesized Drosophila populations. Journal of Comparative and Physiological Psychology, 52, 304-308.

Hirsch, J., \& Erlenmeyer-Kimling, L. (1962). Studies in experimental behavior genetics: IV. Chromosome analyses for geotaxis. Journal of Comparative and Physiological Psychology, 55, 732-739.

Hirsch, J., \& Ksander, G. (1969). Studies in experimental behavior genetics: V. Negative geotaxis and further chromosome analysis in Drosophila melanogaster. Journal of Comparative and Physiological Psychology, 67, 118-122.

Kessler S., \& Kraemer, H. C. (1975). Gene substitutions and geotaxis in Drosophila melanogaster. Journal of Comparative and Physiological Psychology, 89, 274-278. 
Kojima, K., Gillespie, J., \& Tobari, Y. N. (1970). A profile of Drosophila species' enzymes assayed by electrophoresis: I. Number of alleles, heterozygosities and linkage disequilibrium in glucosemetabolizing systems and some other enzymes. Biochemical Genetics, 4, 627-637.

Lewin, B. (1994). Genes V. New York: Oxford University Press.

Lindsley, D. L., \& Zimm, G. G. (1992). The genome of Drosophila melanogaster. San Diego: Academic Press.

Lofdahl, K. L., Hu, D., Ehrman, L., Hirsch, J., \& Skoog, L. (1992). Incipient reproductive isolation and evolution in laboratory Drosophila selected for geotaxis. Animal Behaviour, 44, 783-786.

McClearn, G. E. (1967). Genes, generality and behavioral research. In J. Hirsch (Ed.), Behavior-genetic analysis (pp. 307-321). New York: McGraw-Hill.

McKechnie, S. (1988). [High-geotaxis line fixed for $A d h^{\mathrm{S}}$, low-geotaxis line fixed for $\left.A d h^{\mathrm{F}}\right]$. Unpublished raw data.

McMillan, P. A., \& McGuire, T. R. (1992). The homeotic gene spineless-aristapedia affects geotaxis in Drosophila melanogaster. Behavior Genetics, 22, 557-573.

Murphy, R. W., Sites, J. W., Buth, D. G., \& Haufler, C. H. (1990). Proteins: I. Isozyme electrophoresis. In D. M. Hillis \& C. Moritz (Eds.), Molecular systematics (pp. 45-126). Sunderland, MA: Sinauer Associates.

Pyle, D. W. (1978). A chromosome substitution analysis of geotactic maze behavior in Drosophila melanogaster. Behavior Genetics, 8, 53-64.

Rice, W. R., \& Salt, G. W. (1990). The evolution of reproductive isolation as a correlated character under sympatric conditions: Experimental evidence. Evolution, 44, 1140-1152.

Richmond, R. C. (1984, February 1). [Letter to Jerry Hirsch, with data and photographs]. In J. Hirsch Papers, University of Illinois Archives, No. 15/19/22.

Ricker, J. P. (1984). Selection and hybrid analysis of Drosophila melanogaster for differences in a measure of geotaxis. Unpublished master's thesis, University of Illinois, Urbana-Champaign.

Ricker, J. P., \& Hirsch, J. (1985). Evolution of an instinct under long-term selection for geotaxis in domesticated populations of Drosophila melanogaster. Journal of Comparative Psychology, 99, 380-390.

Ricker, J. P., \& Hirsch, J. (1988a). Genetic changes over 500 generations in lines of Drosophila melanogaster selected divergently for geotaxis. Behavior Genetics, 18, 13-25.

Ricker, J. P., \& Hirsch, J. (1988b). Reversal of genetic homeostasis in laboratory populations of Drosophila melanogaster under long-term selection for geotaxis and estimates of gene correlates: Evolution of behavior-genetic systems. Journal of Comparative Psychology, 102, 203-214.

Sinclair, J. D. (1992). Reply to commentators. Behavior Genetics, 22, 35-42.

Singh, R. S., Hickey, D. A., \& David, J. (1982). Genetic differentiation between geographically distant populations of Drosophila melanogaster. Genetics, 101, 235-256.

Stoltenberg, S. F. (1992). Allozyme monomorphism and an allozyme associated with geotaxis in evolved laboratory populations of Drosophila melanogaster. Unpublished master's thesis, University of Illinois at Urbana-Champaign.

Stoltenberg, S. F., \& Hirsch, J. (1994). Evidence for a Y chromosome effect on geotaxis. Drosophila Information Service, 75, 160-161.

Tully, T., Zawistowski, S., \& Hirsch, J. (1982). Behavior-genetic analysis of Phormia regina: III. A phenotypic correlation between the central excitatory state (CES) and conditioning remains in replicated $\mathrm{F}_{2}$ generations of hybrid crosses. Behavior Genetics, 12, 181-191.

Wahlsten, D. (1990). The insensitivity of the analysis of variance to heredity-environment interaction. Behavioral and Brain Sciences, 13, 109-161. 
Stoltenberg, Hirsch, \& Berlocher, Journal of Comparative Psychology 109 (1995)

Wahlsten, D. (1992). Betwixt gene and behavior (Commentary on the paper by J. D. Sinclair). Behavior Genetics, 22, 11-14.

Weir, B. S. (1990). Genetic data analysis. Sunderland, MA: Sinauer Associates. 